\title{
Efficacy and Safety of Artemether- Lumefantrine, Artesunate-mefloquine Vs Dihydroartemisinin- piperaquine in the Treatment of $P$. Falciparum Malaria in Cameroon
}

Theresia Njuabe Metoh ( $\nabla$ njuabe@yahoo.fr)

University of Bamenda https://orcid.org/0000-0003-3010-4380

\section{Roger Moyou-Somo}

The University of Yaounde I

Philip Fon-Gah

UBa: University of Bamenda

\section{Xia Zhou}

NIPD: National Institute of Parasitic Diseases

\section{Ernest Tambo}

Université des Montagnes FSS: Universite des Montagnes Faculte des Sciences de la Sante

\section{Jun-Hu Chen}

NIPD: National Institute of Parasitic Diseases

\section{Xiaonong Zhou}

NIPD: National Institute of Parasitic Diseases

\section{Research}

Keywords: Artemether-lumefantrine, Artesunate-mefloquine, Dihydroartemisinin-piperaquine, multidrug-resistant, safety, uncomplicated malaria

Posted Date: January 18th, 2021

DOI: https://doi.org/10.21203/rs.3.rs-145331/v1

License: (c) (1) This work is licensed under a Creative Commons Attribution 4.0 International License. Read Full License 


\section{Abstract}

Background: Artemisinin-based Combination Therapies (ACTs), have been reported to be effective against multidrugresistant Plasmodium falciparum. There are controversies in the choice of the type of ACTs to be used at hospitals settings and health centres. This is mostly determined by the cost and less side effects reported for each drug leading to an over prescription and consumption of some ACTs compared to others and consequently to drug resistance. This situation highlight the importance for constant monitoring of ACTs efficacy to provide a guideline to the National Malaria Control Program (NMCP) in readjusting treatment policy when these ACTs are no longer effective and safe in the management of uncomplicated malaria. ACTs including Malacur ${ }^{\circledR}$ (DHAP), artequin ${ }^{\circledR}$ (ASMQ) and cofantrine ${ }^{\circledR}(\mathrm{AL})$, have been reported to be effective against multidrug-resistant Plasmodium falciparum malaria in many countries including Cameroon and the efficacy may have another profile as the consumption increases in the treatment of any fever cases as malaria observed in meso to high malaria endemic countries.

Methods: This open-label, randomized clinical trial was conducted from October 2012 to March 2013 at the Cameroon Development Corporation (CDC) health facilities in Cameroon. Patients who had P. falciparum monoinfection were randomized to receive Malacur ${ }^{\circledR}\left(\right.$ DHAP), artequin ${ }^{\circledR}(A S M Q)$ and cofantrine ${ }^{\circledR}(A L)$ in the treatment of malaria in a high malaria transmission area. Out-patients from the clinics and hospital of the Cameroon Development Corporation having amongst other criteria, a pre-treatment parasite density of $\geq 2000 \mathrm{LL}^{-1}$ of blood were enrolled for the study following the WHO protocol. Informed Consent was obtained from the parents/guardians of the participants and the drugs were given on days 0, 1 and 2. Each patient was followed up to day 42 . Blood slides and filter paper samples for Polymerase Chain Reaction (PCR) were collected on days D0, D1, D2, D3, D14, D21, D28, and D42 post treatment.

Findings: Results showed that of the one thousand five hundred and fifty five (1555) patients screened, two hundred and sixteen (216) meet the enrolment criteria, but 207 completed the trial. Baseline characteristics were similar in all three treatment groups. All of these participants were included in the per- protocol (PP) analysis. The overall crude adequate clinical and parasitological response (ACPR) were 84\% (95\%IC; 83.3- 84.7), 86.9\% (95\% IC; 86.1-87.7\%), 92.1\% (95\% IC; 91.9-92.3\%) in AL, ASMQ, and DHAP arms respectively. The PCR-corrected cure rates were generally higher than crude cure rates. The AL, ASMQ and DHAP PCR-corrected percentage cure rates were 95.6\% (95\% IC; 95.396.0\%), 97.1\% (IC95\%; 96.8-97.4\%) and 99.3\% (IC95\%; 99.1-99.6\%) respectively with a significantly higher cure rate(p < 0.01) recorded for DHAP comparable to ASMQ and AL. The success rates, PCR-corrected for AL on D1, D2, D3, D7, D14, D28 and D42 were 88.90\%, 99.97, 99.99, 100, 100, 97.10 and $94.20 \%$, respectively. The success rates for the ASMQ group were $99.60,99.93,99.96,100,100,97.10 \%$ and $97.10 \%$, respectively. The success rates for the DHAP group were $99.86,99.98,100,100,100,100,98.60$ and $100 \%$ respectively. Parasite clearance time was shorter in the DHAP group $28.7 \pm 9.64$ hours as compare with $\mathrm{AL}, 34.33 \pm 18.40$ hours $(\mathrm{Cl} 95 \% 3.25-8.08 \otimes \mathrm{P}=0.0001)$ and $\mathrm{ASMQ}$ $39.7 \pm 21.4$ hours $(\mathrm{Cl} 95 \%$ 7.34-14.66هP=0.0001). The mean fever clearance times were 12.57, 11.25 and 11.02 hours, for AL, ASMQ and DHAP respectively. The DHAP and ASMQ exhibited marked antigametocyte activity, with a gametocyte clearance time of 48 and 51.0 hours respectively. The adverse reaction common to the three groups of treatment were cough, weakness, abdominal pain, loss of appetite. There were no major adverse reactions in the DHAP group. However, mild but higher frequency of abdominal pain, loss of appetite, vomiting and pruritus was observed in the AL group while mild hallucination and dizziness occurred only in the ASMQ group. It was equally observed that AL, ASMQ and DHAP have an impact in the improvement of Haematocrit rate.

Conclusion: This finding provide evidence that DHAP is the most efficacious, safe and well tolerated ACT compared to ASMQ and AL. Its use is therefore recommended as alternative treatment of malaria in Cameroon and in meso to 
high malaria endemic countries in general.

Trial registration This study is a randomized controlled trial approved by Cameroon National Ethics Committee and retrospectively registered with controlled-trials.com on the 28/11/2016 at the

website: https://clinicaltrials.gov/ct2/show/NCT02974348 with the registration number NCT02974348.

\section{Background}

Malaria continues to be one of the greatest causes of morbidity and mortality in the world [1]. A global annual estimate of 300-500 million of clinical cases and mortality in the range of 1-2 million are attributed to malaria, $92 \%$ of which occurs in Sub-Saharan Africa [2]. In Cameroon, malaria remains the number one public health problem with more than one million five hundred cases and three thousand, one hundred and sixty two deaths in health facilities per year. Overall, $45-50 \%$ of consultations, $23 \%$ of hospitalizations and $35 \%$ of deaths among children under 5 years are attributed to malaria [3]. Following the recommendation of World Health Organization (WHO), for the management of malaria, the National Malaria Control Programme (NMCP) and all scientific community, advocates prompt and adequate treatment as an essential measure to reduce the morbidity and mortality arising from the disease [4,5]. In Cameroon, prior to 2002, chloroquine (CQ) and sulfadoxine-pyrimethamine (SP) were the first and second line antimalarial drugs respectively [6,7], but, there was great concern on the increasing reports of widespread resistance of malaria parasite to these commonly available antimalaria drugs in Cameroon. This was revealed by marked decline of the efficacy of these drugs with $67 \%$ clinical failure for CQ alone [8]. A series of randomised, open controlled trial conducted in South Cameroon revealed $10.2 \%$ and $13.6 \%$ clinical failures for amodiaquine (AQ) and SP, respectively, but no treatment failure for SP+AQ combination $[9,10]$ while other studies conducted in Guinea Savannah showed decline rates of these antimalarials drugs [11]. To overcome this problem, both the WHO and NMCP advocated a change to use of Artemisinin-based Combination Therapy (ACTs) as artemisinins have been found to be the most potent antimalarial drugs and they have an excellent safety profile to ensure effective case management [12]. The principle advanced is that the probability of resistance developing simultaneously to two drugs with independent mechanisms of action is extremely low [13], but studies to ensure that current regimens optimal are few. ACTs are most preferred for their enhancement of efficacy and their potential to lower malaria incidence and the rate at which resistance emerges and spread [14,15]. Due to their rapid clearance time, treating early cases of uncomplicated malaria with ACTs may prevent its progression to severe malaria with consequent reduction in severe cases and malaria mortality rate. The NMCP of Cameroon adopted amodiaquine plus artesunate (AQAS) and artemether plus lumefantrine (AL) for the treatment of uncomplicated malaria in 2004, when there was no local data in support of the policy [16]. In a series of sub-trials to constitute a database of anti-malarial drug efficacy in Cameroon, AQ was proposed to be the most rational partner of artesunate [16,17], likewise, a single arm study provided preliminary evidence of safety and efficacy of AQ+AS [16] but with low statistical power to detect rare events and no Polymerase Chain Reaction (PCR) corrections to distinguish re-infection from recrudescence. The AL dominates the antimalarial market, comprising 77\% of the 331 million ACT treatments used in 2012 [18]. and in previous studies, AL has shown to be highly effective and safe when the twice daily doses (total of six doses) are administered under supervision [17]. However, there are concerns that six doses of AL over three days may reduce compliance [19] and trials comparing it to order ACTs are few [20]. The AS+AQ combination is less expensive and subsidized in Cameroon and it is believed that its cure rate may be lower than that of AL because of parasite resistance to $A Q$ and as such, its inclusion in ACTs is likely going to fail I future [21]. In addition, the transient side effects of $A Q$ may lead to poor compliance and subsequent decline in AQ efficacy [9, 17]. In South East Asia, threeday artesunate-mefloquine treatment is generally the preferred treatment for uncomplicated malarial infection [13]. The Dihydroartemisinin-piperaquine combination as single daily dose has proved to be well tolerated and highly 
effective against uncomplicated falciparum malaria in southeast Asia [22,23] and in Eastern Africa 2007[19,24,25], but very few clinical trials comparing this anti-malarial drug to several ACTs have been conducted in central Africa on children between 0-5 years. However, a randomized non-inferiority open trial has been carried out to compare efficacy and tolerability of DHAP to that of $A L$, in the treatment of uncomplicated falciparum malaria in Cameroon, Ivory Coast and Senegal between 2006 and 2008, but this involves all age groups including 28 days follow-up $[26,27]$. Although ACTs are reported to be highly effective for the management of $P$. falciparum malaria in Africa, reduced clearance rates of parasites from the blood of ACT-treated patients in western Cambodia now raise concerns regarding the emergence and the spread of artemisinin resistance worldwide $[28,29]$. Therefore, monitoring these antimalarial drugs sensitivity is essential to ensure that treatment protocol remain effective. Timely and reliable data from this therapeutic efficacy study will provide a guideline to the NMCPs to detect changing patterns of resistance and make informed decisions on clinical case management by revising treatment guidelines accordingly. Therefore in order to determine the comparative efficacy and adverse effects profile of ACTs in Cameroon, this study aimed at carrying out an open randomised comparative clinical trial of the combination of oral arthemether plus lumefantrine and artesunate plus mefloquine (ASMQ), versus dihydroartemisinine plus piperaquine (DHAP) combinations in the treatment of uncomplicated malaria. If it is demonstrated that DHAP is an effective antimalaria drug in Cameroon, with fewer adverse effects in comparison to ASMQ and AL, it may be an alternative treatment available to the Cameroon Government, with the advantages of being co-formulated as compare to other ACTs. Therefore, the specific objectives of our study are (i) to evaluate the therapeutic efficacy of AL and ASMQ versus DHAP combination in children aged between 6 months to 5 years using the WHO in vivo test extended to 42 day-follow-up period, (ii) to determine the safety and tolerability of those ACTS in the treatment of acute uncomplicated malaria, (iii) to estimate the asexual parasites and gametocytes carriage in each treatment group and their clearance time during treatment, (iv) to assess the clearance of fever, and (v) to determine the parasitological and the hematologic response to treatment.

\section{Materials And Methods}

Study site: The study site is located in the South West Region of Cameroon and include CDC health facilities distributed across different divisions namely the Fako division, Meme Division, Manyu Division, Kupe-Maneguba Division. Annual mean temperature in the South West region is $24.5^{\circ} \mathrm{C}$ suitable for the proliferation of anopheles vectors $[30,31]$ with equatorial climate having a total annual rainfall that ranges from $2600 \mathrm{~mm}$ in the southern low plateau to over $10.000 \mathrm{~mm}$ in Debuncha. The Cameroon Development Corporation (CDC) is located within a high malaria transmission area, hence its suitability for trials of this nature. Anopheles gambiae accounts for the highest transmission as compare with An. funestus and An. Nili. [30]Malaria transmission occurs both in the dry and rainy season with high rate intensities in the dry season and an Entomologic Inoculation Rate (EIR) of 287 infective bites/person/year [31]. The CDC is hosting rural community in South West which is about $30 \mathrm{~km}$ south of Buea town and $45 \mathrm{~km}$ from Douala, Cameroon. Houses are grouped into camps which are structured buildings provided by the company to accommodate the workers and their families. The CDC Company has a head quarter in Limbe town headed by a Director, a reference hospital and satellite clinics. There are also Primary Health Centres and hospitals, Private Clinics, mission and government health facilities, clinic and an aid post found at the level of each camp.

\section{Open clinical trial and grouping:}

During the open clinical trial, patients were recruited in the outpatient pediatric unit of reference hospital and satellite clinics and follow up for 42 days, between October 2012 and March 2013. The recruit criteria for entry into the open trial include age stratified into 6 months- 5 years, absence of severe malnutrition by clinical examination and by 
measurement of weight against the height, monoinfection with $P$. falciparum parasitaemia in the range of 2000 to 200,000 asexual parasites per $\mu \mathrm{L}$ of blood, presence of axillary temperature $\geq 37.5^{\circ} \mathrm{C}$ and/or history of fever in the preceding $24 \mathrm{hrs}$, informed consent by parent/guardian, ability to come for the stipulated follow-up visits and, easy access to the health facility. The exclusion criteria for the study cases were: presence of general danger signs such as: Not able to drink or breastfeed, vomiting everything, recent history of convulsion, lethargic or unconscious state, unable to sit or stand up and use of any drug known to influence cardiac function (e.g., Halofantrine) within 4 weeks before screening. Also excluded were those showing signs of severe and complicated falciparum malaria, namely, cerebral malaria, severe anaemia (Hct $<15 \%$ at day 0 ), febrile conditions caused by diseases other than malaria and History of allergy to study drugs.

\section{Sample size estimation}

An expected treatment failure rate of $5 \%$ was assumed for all the three drugs evaluated including malacur ${ }^{\circledR}$ (dihydroartemisinin plus piperaquine), cofantrine ${ }^{\circledR}$ (arthemether plus lumefantrine), Artequin ${ }^{\circledR}$ (artesunate plus mefloquine) , with a $95 \%$ confidence level and a precision of $5 \%$, a sample size of 60 was targeted per drug. An additional $20 \%$ was added to ensure that the sample size would be achieved after the exclusion of patients due to loss to follow-up or withdrawal. A total of 72 patients per drug would be required making a total of 216 participants for this trial.

\section{The study design}

The study design essentially followed the WHO guidelines for the assessment and monitoring of antimalarial drug efficacy with an extension of follow-up until day 42 [32]. It is a randomized comparative clinical trial of three days regimen of a combination of malacur ${ }^{\circledR}$ (dihydroartemisinin plus piperaquine), cofantrine ${ }^{\circledR}$ (arthemether plus lumefantrine), Artequin ${ }^{\circledR}$ (artesunate plus mefloquine) for efficacy, safety and tolerability that was carried out in CDC estates, Tiko health district. Patients who meet the inclusion criteria were enrolled in the study after informed concern were obtained from the parents/guardians following explanatory notes. Day 0 was the day of screening, clinical assessment, initial malaria smears and taking of blood for hematocrit assessment. The participants in each arm also had their blood taken for PCR analysis. They were allocated to one of the three treatment groups and given the first dose of either malacur ${ }^{\circledR}$, or cofantrine ${ }^{\circledR}$ or artequin ${ }^{\circledR}$ on day 0 . After drug administration on day 0 , the patients were asked to return on days 1 and 2 to complete the drug regimen and for clinical assessment. Patients were asked to return to the study centre on days $1,2,3$, and 7 and then weekly until day 42 . If the patients did not return for the scheduled visits or missed an appointment, they were seen at their homes by the study staff. Blood smears were prepared and body temperature were recorded at each visit. In case of any symptoms consistent with malaria, patients were advised to return to the centre immediately.

\section{Treatment, Randomization, and Blinding:}

Patients were randomly assigned to receive one of three oral therapies: AL or ASMQ or DHAP on days 0,1 , and 2. AL tablets were a fixed combination, each containing $20 \mathrm{mg}$ of artemether and $120 \mathrm{mg}$ of lumefantrine. AL was administered according to body weight (5-14 kg: one tablet; 15-24 kg: two tablets; 25-34 kg: three tablets;>35 kg: four tablets) as six consecutive doses: The first dose at diagnosis and the second dose eight hours later on Day 0 , and then two doses at 12 hours intervals for the subsequent two days. Patients allocated to the three-day treatment with ASMQ or DHAP were given their treatment three times, at an interval of 24 hours $(0 \mathrm{~h}-24 \mathrm{~h}-48 \mathrm{~h})$.

Randomization codes were computer-generated by an offsite investigator and provided to a study nurse responsible for treatment allocation. All other study personnel were blinded to the treatment assignments, and patients were not 
informed of their treatment regimen. The drugs were administered under medical supervision and treated patients were observed for $60 \mathrm{~min}$. If vomiting occurred within $30 \mathrm{~min}$ of administration of the drug, the same dose was repeated. However, if it occurred 30-60 min, half the dosage was given again. Further vomiting entailed protocol violation. The patient was excluded and rescue treatment with quinine was used. Any use of concomitant medications (including acetaminophen i.e., paracetamol) were documented in the Case Report Form (CRF). Participants were also given appointment card for days 1, 2, 3, 7, 14, 21, 28, 35 and 42 for clinical examination and blood smears. Blood was taken on filter papers on each of these visits. They were also asked to return to the clinic on days other than these if they developed any additional complaints, or any change in their condition compared to preadministration of the drug. Additional efforts were made by the field workers to locate home address of each patient for home visit in case if a patient did not report at the hospital or clinics for the scheduled visit.

\section{Laboratory investigations and follow-up:}

Clinical assessment and parasite density counts were performed on days $0,1,2,3,7,14,21,28,35$ and 42 or on any day of recurrent malaria infection. Patient's rectal temperature were measured immediately before and then every 12 $\mathrm{h}$ after starting treatment until their temperature was $<38^{\circ} \mathrm{C}$ for two consecutive days. Rectal body temperature was recorded using a digital electronic thermometer. At screening prior to enrolment, thick and thin blood films were examined. A second, Giemsa stained thick film was examined with a binocular microscope with an oil immersion objective lens to quantify the parasitaemia. Parasite density was measured counting the number of asexual parasites against a number of leukocytes in the thick blood film, based on a putative count of 8000 leukocytes per microlitre of blood. The number of asexual parasites was counted against 200 leukocytes using a hand tally counter. If $P$. falciparum gametocytes were seen, a gametocyte count was performed against 1000 leukocytes (WHO/MAL/82.988).

The classification of the therapeutic outcome was done according to the WHO protocol [32]. The primary endpoint was the 28-day and 42-day cure rates and was defined as proportion of patients with adequate clinical and parasitological response (ACPR) after 28 and 42 days of follow-up. Absence of parasitemia until day 28 and day 42 irrespective of axillary temperature was categorized as an adequate clinical and parasitological response (ACPR). The primary efficacy out-comes were analysed using both the intention-to-treat (ITT) and per-protocol (PP) data sets. The ITT population was made up of all patients who received at least one dose of study drug. The PP analysis included patients who adhered to the protocol until attainment of an end point or 42 days post-treatment. Uncorrected efficacy was determined by dividing the number of treatment failures in each study arm by the total number of participants classified as either adequate clinical and parasitological response or treatment failure in that arm. Parasite clearance rates on day 2 and day 3 of follow-up were examined to evaluate artemisinin delayed response. PCR-adjusted cure rates between treatments were compared using $\chi 2$ test as well as the odds ratios for likelihood of cure with $95 \%$ confidence intervals (Cls). Secondary endpoints were early treatment failure (ETF), late clinical failure (LCF), late parasitological failure (LPF), adverse events (clinical and laboratory abnormalities), anaemia (Haematocrit < 30\%), clearance rate of fever and parasitaemia, and gametocyte carriage. Parasite and fever clearances were assessed on days 1,2 , and 3.

Fever clearance time (FCT) was defined as the time in hours from drug administration until the rectal temperature decreased to $<38^{\circ} \mathrm{C}$ and remained so for 48 hours. Parasite clearance time (PCT) was the time in hours from starting treatment until the asexual parasite count fell below detectable levels in thick blood films. Drug tolerability was assessed clinically. Adverse event was defined as a sign or symptom not present on day 0 , but which occurred during follow-up, or was present on day 0 but became worse during follow-up regardless as to whether it was related to the medication. Serious adverse events were defined according to International Conference on Harmonization (ICH) 
guidelines. Adverse events were recorded by the physician at each drug administration. Adverse events, unsatisfactory therapeutic effects, loss of patient follow-up, patient non-compliance or consent withdrawal or withdrawal as a result of treatment failure, were criteria for discontinuation. Treatment efficacy was determined based on parasitological cure rates by the times to parasite and fever clearance; and from the proportion of patients without gametocyte. Recrudescence denoted clinical recurrence of malaria after the initial clearance of parasite from the circulation. Parasite reappearance was interpreted as either true recrudescence or a new infection [33]. Clinical therapeutic outcomes were adjusted by genotyping the $P$. falciparum merozoite surface protein 2 ( $m s p 2$ ), merozoite surface protein 2 ( $m s p 2$ ) and glutamate rich protein (glurp) on admission (Day 0 ) and any day of infection recurrences (Day 7, 14, 21, 35, 28 and D42). Under this assessment, only parasitaemia that was confirmed by PCR as recrudescence was considered as treatment failure and conversely, was considered as new infection and counted as the ACPR.

\section{Data management and statistical analysis}

Data generated in patient's case record forms were entered in excel and data analysis performed using SPSS statistical analysis software package version 17. Descriptive analysis was done and differences in proportions of treatment outcome were compared using Chi-square or Fisher exact test. An independent samples t-test was applied for continuous variables. All reported p-values are two sided without adjustment for multiple testing and were considered statistically significant if below 0.05 . Data on patients that were excluded for different reasons and those that were loss to follow up were not considered in the final analysis. PCR-adjusted cure rates between treatments were compared using $\chi^{2}$ test as well as the odds ratios for likelihood of cure with $95 \%$ confidence intervals (Cls).

\section{Ethics and good clinical practice (GCP)}

This study protocol, the CRF, information sheet and concern form were reviewed and approved by the National ethic committee of Cameroon who gave written permission to carry out the study. The medical board in charge of hospitals and clinics as well as the administrative authorities of the CDC Company also consented to the conduct of the study and gave administrative clearance. The study was carried out in compliance to the protocol, in accordance with the principles laid down by the World Health Assembly of 1975 on Ethics in Human experimentation and the Helsinki Declaration. The study adhered to Good Clinical Practices (GCP) [34], and conformed to the TDR Standard Operating Procedures (SOP) approved by IRB of the National Institute of Parasitic Diseases, Chinese Centre for Disease Control and Prevention.

\section{Results}

\section{Participant characteristics at baseline}

The study was conducted between October 2012 and March 2013, with a total of 1555 patients screened because they complained of symptoms suspected to be of malaria and had not taken any antimalarial medication within the previous days. The study profile is shown in figure 1. At enrolment, the three treatment groups had similar demographic and clinical characteristics. Overall, 225 patients fulfilled the criteria for enrolment but 9 where either residents inaccessible areas to the study team or were on transit in the Cameroon Development Corporation company. A total 216 were randomized consisting of 72 patients on AL, 72 patients on ASMQ and 72 on DHAP. However, one patient who was lost during follow up, was reported dead in the group of AL. Two other patients in the same group of AL, 4 in ASMQ and 2 in the DHAP group defaulted as a result of withdrawal or loss to follow-up and/or protocol violation. The demographic and clinical characteristics of the patients are shown in table 1. 
Table 1

Demographic and clinical characteristics of patients per treatment group, at enrolments (Day 0) CAMEROON DC, 2012-2013.

\begin{tabular}{|c|c|c|c|c|c|}
\hline Characteristics & $\mathrm{AL}(\mathrm{N}=72)$ & ASMQ (N=72) & $\operatorname{DHAP}(\mathrm{N}=72)$ & $\begin{array}{l}P \\
(1)\end{array}$ & $\begin{array}{l}P \\
(2)\end{array}$ \\
\hline Mean Age (months) SD & $32.97(16.4)$ & $37.90(16.7)$ & $42.8(14.6)$ & 0.15 & 0.15 \\
\hline Mean Weight (Kg) SD & $12.9(2.8)$ & $13.7(3.1)$ & $14.4(2.6)$ & 0.47 & 0.19 \\
\hline Sex-ratio Male / Female & $1.8(44 / 28)$ & $1(36 / 36)$ & $0.8(32 / 40)$ & & \\
\hline \multirow[t]{2}{*}{ Mean Temperature $\left({ }^{\circ} \mathrm{C}\right) . \mathrm{SD}$ (Range) } & $38.1(1.1)$ & $38.4(1.0)$ & $38.2(1.0)$ & 0.50 & 0.13 \\
\hline & $(36-41)$ & $(36.03-40.08)$ & $(36.7-40.6)$ & & \\
\hline \multirow{2}{*}{$\begin{array}{l}\text { Mean temperature } \geq 38^{\circ} \mathrm{C}\left({ }^{\circ} \mathrm{C}\right) . \mathrm{SD} \\
\text { (Range) }\end{array}$} & $39.1(1.0)$ & $38.97(0.8)$ & $38.8(0.9)$ & 0.51 & 0.45 \\
\hline & $(38-41)$ & $(38-40.8)$ & $(38-39.8)$ & & \\
\hline \multirow[t]{2}{*}{ Mean temperature $<38$ SD (Range) } & $37.3(0.45)$ & $37.4(0.4)$ & $37.4 \llbracket 0.4 \rrbracket$ & 0.70 & 0.90 \\
\hline & $(36-37.9)$ & $(36.3-37.9)$ & $(36.7-37.9)$ & & \\
\hline \multirow[t]{2}{*}{ Proportion $\geq$ to $40^{\circ} \mathrm{C} \mathrm{SD}$ (range) } & $40.3 \otimes 0.3 \rrbracket$ & $40.2 \otimes 0.3 \rrbracket$ & $40.17 \bowtie 0.2 \rrbracket$ & 0.23 & 0.90 \\
\hline & $(40-41)$ & $(40-40.2)$ & $(40-40.6)$ & & \\
\hline \multirow{2}{*}{$\begin{array}{l}\text { Geometrical mean Parasite density } 邓 p / u l \rrbracket \\
S D\left({ }^{*} \mathrm{Cl} \% 95\right)\end{array}$} & 27,061 & 25135 & 19002 & 0.09 & 0.65 \\
\hline & $(-6074-22192 \rrbracket$ & $(-13627-1747 \rrbracket$ & $\begin{array}{l}(-6074- \\
22192 \rrbracket\end{array}$ & & \\
\hline \multirow{2}{*}{$\begin{array}{l}\text { Geometrical mean } \geq 100000(\mathrm{P} / \mathrm{ul}) \\
\text { SD(Cl\%95) }\end{array}$} & 198849.8 & 185500.3 & 130670.7 & 0.5 & 0.2 \\
\hline & $\begin{array}{l}(-1840.5- \\
138198.6)\end{array}$ & $\begin{array}{l}(-30969.6- \\
140628.8)\end{array}$ & $\begin{array}{l}(-1840.5- \\
38198.6)\end{array}$ & & \\
\hline Mean Hematocrite $\llbracket \% \bigotimes S D(P>0.05)$ & $33.2 \varangle 6.0 \rrbracket$ & $32.8 \varangle 6.0 \rrbracket$ & $33.8 \otimes 5.9 \rrbracket$ & 0.56 & 0.13 \\
\hline \multirow[t]{2}{*}{ Anemia between $15 \%$ et $29 \%$} & $23 / 72$ & $28 / 72$ & 19/72 & & \\
\hline & $(31.9 \%)$ & $(38.9 \%)$ & $(28.4)$ & & \\
\hline
\end{tabular}

* Confidence interval at 95 \% (binomial exact method). (1) Compare DHAP vs AL. (2) Compare DHAP vs ASMF.

The 28 days follow up was completed for 209 (96.7\%) patients and 207 (95.8\%) subjects were evaluable for primary and secondary endpoint on day 42 consisting of 69/72 (95.8\%) children treated with AL, 68/72 (94.4\%) children treated with ASMQ and 70/72 (97.2\%). Of the patients recruited, $38.9 \%(28 / 72)$ on AL, $50.0 \%(36 / 72)$ on ASMQ and $55.5 \%(40 / 72)$ on DHAP were female. The mean age was $32.97 \pm 16.41$ months for AL, $32.97 \pm 16.41$ for ASMQ and $42.78 \pm 14.57$ months for DHAP with no significant $(P=0.15)$ difference in the mean age between the treatment groups. The mean weight was $12.89 \pm 2.76 \mathrm{~kg}, 90 \pm 16.68 \mathrm{Kg}, 14.38 \pm 2.64 \mathrm{Kg}$ for AL, ASMQ and DHAP treatment groups respectively. There was no statistical significant difference between the treatment group relative to the mean weight $(P>0.05)$. The geometrical mean parasitaemia was comparable between the treatment groups: 27,061 parasites $/ \mu \mathrm{L}$ for AL and 19002 parasites/ $\mu \mathrm{L}$ for DHAP (AL vs DHAP CI95\% (-6074-22192) and 25135 parasites/ $\mu \mathrm{L}$ for ASMQ, (AL vs ASMQ CI95\% (-13627-1747). The geometrical mean parasitaemia was higher in AL group compared with ASMQ and DHAP groups, but the differences were not significant (AL vs DHAP - $P=0.09$; AL vs ASMQ $-P=0.65$ ). At the time 
of admission, $45.83 \%$ (33/72) of patients on AL, 66.67\% (48/72) of patients on ASMQ and 55.55\% (40/72) on DHAP were febrile $\left(T \geq 38^{\circ} \mathrm{C}\right)$. A total of 12 patients showed a parasite density greater or equal to $100000 \mathrm{P} / \mathrm{uL}$ including 5 patients with the mean parasite density of $198849.8 \mathrm{P} / \mathrm{uL}$ ( Cl 95\% -1840.5-138198.6) in the AL group,3 patients with the mean parasite density of 185500.3 P/uL (IC\% -30969.6-140628.8) in the ASMQ group and 4 with the mean parasite density of $130670.7 \mathrm{P} / \mathrm{uL}$ ( $\mathrm{Cl}$ 95\%-1840.5-138198.6) in the DHAP group. No statistical difference $(P>0.05)$ was observed between the groups (table 1). Likewise, 2 patients in the AL group and one in the ASMQ had a parasite density count of greater or equal to $200000 \mathrm{P} / \mathrm{uL}$ of blood; they were enrolled because they had no sign and symptoms of severe malaria at inclusion. Values of vital signs (pulse rate and RR) evaluated at enrolment and during the study course were within the expected ranges. At enrolment, the pulse rates ranging from 108 to $115 \mathrm{~b} / \mathrm{m}$ and RR from 24 to 26 . There was no tachycardia and dyspnoea. The haematocrit values were between $36 \%$ to $38 \%$. At base line 91 of 216 enrolled patient had rectal body temperature below $38^{\circ} \mathrm{C}$ with high parasitaemia reflecting a possible self-medication with analgesic before coming to the hospital or clinic.

\section{The clinical and parasitological response to DHAP, ASMQ and AL}

The outcomes of clinical and parasitological response are showed in Table 2. None of the patients in the three different treatment arms showed early treatment failure during the first three days after initiation of treatment. The drugs were tolerated; there was no report of significant Adverse Drug Reaction (ADR). Table 2 shows crude and PCR corrected treatment rates of the test drugs. It was observed in the course of this trial, that parasitaemia consisting of young trophozoites appeared on day 28 and 42 in 18 of the 68 ASMQ group, 22 of the 69 AL group and 12 in the 70 DHAP group. The parasitaemia was associated with increase in body temperature in 6,4 and 3 patients in AL, ASMQ and DHAP treatment group respectively. Blood sample were collected to perform the Polymerase Chain Reaction (PCR) technique in order to infer whether it was as a result of recrudescence or of new infection. 
Table 2

Clinical and parasitological therapeutic outcome after PCR correction

\begin{tabular}{|c|c|c|c|c|c|c|c|c|}
\hline & $\mathrm{AL}$ & & ASMQ & & DHAP & & $P(1)$ & $P(2)$ \\
\hline \multicolumn{9}{|l|}{$\begin{array}{l}\text { Per- } \\
\text { protocol } \\
\text { day } 28\end{array}$} \\
\hline & $\begin{array}{l}\text { Before } \\
\text { PCR } \\
\text { corrections } \\
(\%)\end{array}$ & $\begin{array}{l}\text { After PCR } \\
\text { corrections } \\
(\%)\end{array}$ & $\begin{array}{l}\text { Before } \\
\text { PCR } \\
\text { corrections } \\
(\%)\end{array}$ & $\begin{array}{l}\text { After PCR } \\
\text { corrections } \\
(\%)\end{array}$ & $\begin{array}{l}\text { Before } \\
\text { PCR } \\
\text { corrections } \\
(\%)\end{array}$ & $\begin{array}{l}\text { After PCR } \\
\text { corrections } \\
(\%)\end{array}$ & & \\
\hline ETF & $0(0.0)$ & $0(0.0)$ & $0(0.0)$ & $0(0.0)$ & $0(0.0)$ & $0(0.0)$ & & \\
\hline LCF & $2(2.9)$ & $1(1.4)$ & $4(5.7)$ & $2(2.9)$ & $1(1.4)$ & $1(1.4)$ & & \\
\hline LPF & $7(10.1)$ & $1(1.4)$ & $3(4.3)$ & $0(0.0)$ & $4(5.7)$ & $0(0.0)$ & & \\
\hline $\begin{array}{l}\text { ACPR } \\
\text { (N; } \\
\text { IC95\%)* }\end{array}$ & $\begin{array}{l}60(86.9) \\
\text { (IC95\%; } \\
85.3- \\
88.5 \%)\end{array}$ & $\begin{array}{l}67(97.1) \\
(I C 95 \% \\
96.8- \\
97.4 \%)\end{array}$ & $\begin{array}{l}63(90.0) \\
\text { (IC95\%; } \\
88.8- \\
91.2 \%)\end{array}$ & $\begin{array}{l}68(97.1) \\
\text { (IC95\%; } \\
96.8- \\
97.4 \%)\end{array}$ & $\begin{array}{l}65 \text { (92.9) } \\
\text { (IC95\%; } \\
92.1- \\
93.7 \%)\end{array}$ & $\begin{array}{l}69 \text { (98.6) } \\
(I C 95 \% ; \\
98.4- \\
98.8 \%)\end{array}$ & 0.002 & 0.002 \\
\hline Total & 69 & 69 & 70 & 70 & 70 & 70 & & \\
\hline \multicolumn{9}{|l|}{$\begin{array}{l}\text { Per- } \\
\text { protocol } \\
\text { day } 42\end{array}$} \\
\hline LCF & $4(6.0)$ & $1(1.4)$ & $0(0.0)$ & $0(0.0)$ & $1(1.4)$ & $0(0.0)$ & & \\
\hline LPF & $9(13.0)$ & $3(4.5)$ & $11(16.8)$ & $2(2.9)$ & $5(7.2)$ & $0(0.0)$ & & \\
\hline $\begin{array}{l}\text { ACPR } \\
(\mathrm{N} ; \\
\text { IC95\%)* }\end{array}$ & $\begin{array}{l}56(81.1) \\
(\mathrm{IC} 95 \% \\
78.8- \\
83.4 \%)\end{array}$ & $\begin{array}{l}65 \text { (94.2) } \\
\text { (IC95\%; } \\
93.5- \\
94.9 \%)\end{array}$ & $\begin{array}{l}57 \text { (83.8) } \\
\text { (IC95\%; } \\
81.8- \\
85.8 \%)\end{array}$ & $\begin{array}{l}66 \text { (97.1) } \\
\text { (IC95\%; } \\
96.8- \\
97.4 \%)\end{array}$ & $\begin{array}{l}63 \text { (91.3) } \\
\text { (IC95\%; } \\
90.3- \\
92.3 \%)\end{array}$ & 70 (100) & 0.01 & 0.003 \\
\hline Total & 69 & 69 & 68 & 68 & 70 & 70 & & \\
\hline $\begin{array}{l}\text { ACPR } \\
\text { D7 - } \\
\text { D42 }\end{array}$ & $\begin{array}{l}84 \% \text { (IC } \\
95 \% ; 83.3- \\
84.7 \%)\end{array}$ & $\begin{array}{l}95.6 \% \\
\text { (IC95\%; } \\
95.3- \\
96.0 \%)\end{array}$ & $\begin{array}{l}86.9 \% \\
\text { (IC95\%; } \\
86.1- \\
87.7 \%)\end{array}$ & $\begin{array}{l}97.1 \% \\
\text { (IC95\%; } \\
96.8- \\
97.4 \%)\end{array}$ & $\begin{array}{l}92.1 \% \\
\text { (IC95\%; } \\
91.9- \\
92.3 \%)\end{array}$ & $\begin{array}{l}99.3 \% \\
\text { (IC95\%; } \\
99.1- \\
99.6 \%)\end{array}$ & 0.004 & 0.003 \\
\hline
\end{tabular}

Per-protocol efficacy: proportion of adequate clinical and parasitological response, * Confidence interval at $95 \%$ (binomial exact method); (1) Compare DHAP vs AL; (2) Compare DHAP vs ASMF

The overall crude adequate clinical and parasitological response (ACPR) were 84\% (95\%IC; 83.3- 84.7), 86.9\% (95\% IC; 86.1-87.7\%), 92.1\% (95\% IC; 91.9-92.3\%) in AL, ASMQ, and DHAP arms respectively (table 2). The PCR-corrected cure rates were generally higher than crude cure rates. The AL, ASMQ and DHAP PCR-corrected percentage cure rates were 95.6\% (95\% IC; 95.3-96.0\%), 97.1\% (IC95\%; 96.8-97.4\%) and 99.3\% (IC95\%; 99.1-99.6\%) respectively with a significantly higher cure rate $(\mathrm{p}<0.01)$ recorded in DHAP compared to ASMQ and AL arms (table 2). The success rates, PCR-corrected for AL on D1, D2, D3, D7, D14, D28 and D42 were 88.90\%, 99.97, 99.99, 100, 100, 97.10 and $94.20 \%$, respectively. The success rates for the ASMQ group were 99.60, 99.93, 99.96, 100, 100, $97.10 \%$ and $97.10 \%$, respectively. The success rates for the DHAP group were 99.86, 99.98, 100, 100, 100, 100, 98.60 and 100\% respectively. 


\section{Profile of parasite clearance time (PCT) and temperature clearance time (TCT)}

Parasite clearance was slower in the AL group as compared to the ASMQ and DHAP treatment groups. However, proportions of aparasitaemic patients for the 42-day duration were similar for the AL, ASMQ and DHAP arms on days 7, 14 and 35 (figure 2). Importantly, 99.61\% of DHAP-treated patients and $99.86 \%$ of ASMQ treated patients were aparasitaemic on day 1 and $100 \%$ of patients in DHAP arm were aparasitaemic on day 2 (figure 2).

The median time to parasite clearance for each treatment group is presented in table 3. The mean PCT was shorter in DHAP group $28.7 \pm 9.64$ hours as compared to $\mathrm{AL}, 34.33 \pm 18.40$ hours $(\mathrm{Cl} 95 \% 3.25-8.08 \otimes \mathrm{P}=0.0001)$ and $\mathrm{ASMQ} 39.7 \pm$ 21.4 hours (Cl 95\% 7.34-14.66; $P=0.0001)$.

Table 3

Parasite and temperature clearance

\begin{tabular}{|c|c|c|c|}
\hline Treatment Outcome & $\mathrm{AL}(\mathrm{N}$ total $=72)$ & ASMF ( $\mathrm{N}$ total $=72)$ & DHAP ( $\mathrm{N}$ total $=72)$ \\
\hline Fever clearance Time (hours) & $12.57 \mathrm{~h} \pm 3.50$ & $11.25 h \pm 5.11$ & $11.02 \mathrm{~h} \pm 2.33$ \\
\hline Parasite Clearance time (hours) SD (Cl95\%) & $\begin{array}{l}34.3 \pm 18.4(3.2- \\
8.1 \otimes P=0.0001)\end{array}$ & $\begin{array}{l}39.7 \pm 21.4 \rrbracket 7.3- \\
14.7 \llbracket P=0.0001)\end{array}$ & $\begin{array}{l}28.7 \pm 9.64(3.2- \\
8.1 \otimes P=0.0001)\end{array}$ \\
\hline Positive blood film at D1 (N (\%)) & $18(25)$ & $21(29.2)$ & $14(19.4)$ \\
\hline Positive blood film at D2 (N (\%)) & $11(15.3)$ & $13(18.0)$ & $11(15 . .3)$ \\
\hline Positive blood film at D3 (N (\%)) & $2(2.8)$ & $1(1.4)$ & 0 \\
\hline Withdrawal/loss to follow-up (D1-D7) & 0 & 0 & 0 \\
\hline Withdrawal/loss to follow-up $\varangle \mathrm{D} 7-14 \rrbracket$ & 2 & 0 & 0 \\
\hline LTF $\triangle D 7-14 \rrbracket$ & 0 & 0 & 0 \\
\hline Analysed at D14 & 70 & 72 & 72 \\
\hline $\begin{array}{l}\text { aAdequate clinical and Parasitological } \\
\text { Response (ACPR) Day } 14(\mathrm{~N}, \%)\end{array}$ & $70(100)$ & $72(100)$ & $72(100)$ \\
\hline Analysed at D28 & 69 & 70 & 70 \\
\hline Withdrawal/loss to follow up & 1 & 2 & 2 \\
\hline LTF (D15-28) & 2 & 4 & 1 \\
\hline Late parasitological failure (LPF) (N/total, \%) & 7 & 3 & 4 \\
\hline $\begin{array}{l}\text { aAdequate clinical and Parasitological } \\
\text { Response (ACPR) Day } 28(\mathrm{~N}, \%)\end{array}$ & $\begin{array}{l}60(86.9)(\text { IC95\%; } \\
85.3-88.5 \%)\end{array}$ & $\begin{array}{l}63(90.0)(\text { (IC95\%; } \\
88.8-91.2 \%)\end{array}$ & $\begin{array}{l}65(92.9)(\text { IC95\%; } \\
92.1-93.7 \%)\end{array}$ \\
\hline Analysed at D42 & 69 & 68 & 70 \\
\hline Withdrawal/loss to follow-up & 0 & 0 & 0 \\
\hline Late treatment failure on day 42 & 4 & 0 & 1 \\
\hline Late parasitological failure (LPF) (N/total, \%) & 9 & 11 & 5 \\
\hline $\begin{array}{l}\text { aAdequate clinical and Parasitological } \\
\text { Response (ACPR) Day } 42 \text { (N/total, \%) }\end{array}$ & $\begin{array}{l}56(81.1)(I C 95 \% \\
78.8-83.4 \%)\end{array}$ & $\begin{array}{l}57(83.8)(\text { IC95\%; } \\
81.8-85.8 \%)\end{array}$ & $\begin{array}{l}63(91.3)(\text { IC95\%; } \\
90.3-92.3 \%)\end{array}$ \\
\hline
\end{tabular}


Note: aPCR uncorrected.

Time for Parasite Clearance (PCT) in 72 patients of $A L$ group was determined and it was observed that Parasitaemia completely cleared in 53 patients within $24 \mathrm{hrs}, 7$ cleared in $4 \mathrm{hrs}$ while 12 patients were cleared in $72 \mathrm{~h}$. Time for parasite clearance was $34.33 \pm 18.40$ hours for the AL group. In AL group, the geometric mean parasite density of the 72 enrolled patients on day 0 was 27061.1 which decreased to 2988.4 on D1, therefore giving a percentage success rate of $88.9 \%$. The success rates (using the geometric mean parasite densities) on day 2 and 3 were $88.90 \%$, and 99.97respectively. In ASMQ group, the geometric mean parasite density of the 72 enrolled patients on day 0 was 25135.1 which reduced to 107.0 on D1 therefore giving a percentage success rate of $99.6 \%$ on D1. The success rates on other days, using the geometric mean parasite density, were as follows: D2 (99.93\%), D3 (99.96\%). Hence, parasitaemia was cleared in 45 children within $24 \mathrm{~h}$ (D1), 7 children within 48hrs (D2) and in the remaining 20 children within $72 \mathrm{hrs}$ (D3). Therefore the time to parasite clearance was calculated to be $39.7 \pm 21.4 \mathrm{hrs}$ for the ASMQ group. In the DHAP group, the geometric mean parasite density of the 72 enrolled patients on day 0 was $19,002.4$ which reduced to 26.70 on D1 therefore giving a percentage success rate of $99.86 \%$ on D1. The success rates on other days, using the geometric mean parasite density, were as follows: D2 (99.98\%), D3 (100\%). One child showed a reappearance of parasite on day 28 and one on day 42 associated with high temperature $\left(39-40^{\circ} \mathrm{C}\right)$ and they were given antipyretic and quinine tablets for 7 days since no sign and symptoms of severe malaria demanded referral. Parasitaemia had cleared in 58 children within 24hrs (D1), 3 children within 48 hrs (D2), 11 within $72 \mathrm{hrs.} \mathrm{Most}$ Important, no child had residual parasitaemia on day 3 in DHAP recipients, giving a success rate of $100 \%$ within $72 \mathrm{hrs}$ (D3). Therefore the time to parasite clearance was calculated to be $28.7 \pm 9.64 \mathrm{~h}$. Fever clearance time was shorter in DHAP $(11.02 \pm 0.51 \mathrm{hrs})$ and ASMF $(11.25 \pm 4.7 \mathrm{hrs})$ group as compare to the AL group (12.57 $\pm 4.0 \mathrm{hrs})$

Overall, 122 patients 33 in AL group, 48 ASMQ group and 41 in DHAP group showed a temperature of above or equal to $38^{\circ} \mathrm{C}$ with a mean temperature of $38.13 \pm 1.15^{\circ} \mathrm{C}, 38.44 \pm 1.02^{\circ} \mathrm{C}$ and $38.18 \pm 1.01^{\circ} \mathrm{C}$ for the $\mathrm{AL}, \mathrm{ASMQ}$ and DHAP group respectively with no statistical significant difference $(P>0.05)$ between the treatment group (Table 1).

A total number of 31 patients including 10 in AL group, 13 in ASMQ group and 8 in DHAP group harboured a rectal body temperature of $40^{\circ} \mathrm{C}$ and above with the mean temperature $40.35 \pm 0.32^{\circ} \mathrm{C}$ of in the $\mathrm{AL}$ treatment group; $40.05 \pm$ $0.08{ }^{\circ} \mathrm{C}$ in ASMQ and 40.17 in DHAP group. Overall, there was a significant decrease of these temperature from D0 and throughout the follow period (figure 3 ) without any significant difference $(P>0.05)$ between the treatment groups.

\section{Tolerability and safety}

Tolerability defines the overall profile of adverse drug reactions, whereas safety refers to potentially hazardous adverse drug reactions. In this trial tolerability and safety evaluations were based on measurement of vital signs, physical examination, recording of all adverse events, and haematology analysis. Patients were equally asked not leading questions on their health situation since the last intake of antimalarial drugs and answers were classified as mild, moderate and serious or life threatened.

\section{(1) EFFECT OF TRIAL ANTIMALARIAL DRUGS ON ANEMIA}

AL, ASMQ and DHAP, improved on severe anaemia (HCT < 25\%) in two to four weeks. The increase of HCT was in the order of $2 \%$ to $4 \%$ on D14 and $5 \%$ to $8 \%$ D28, and $8 \%$ to $10 \%$ on D42 as compare to baseline (Table 3 , Figure 4 ). The mean haematocrit improved moderately from day 0 to day 28 by 3 to $5 \%$ in AL group, by 4 to $8 \%$ in ASMQ group and 2 to $5 \%$ in DHAP treatment group. The mean Haematocrit recovery after day 28 was statically significant in all the

Page $12 / 25$ 
treatment groups $(p<0.001)$. The mean Haematocrit value was $33.2 \pm 6.0 \%$ for AL $32.8 \pm 6.0 \%$ for ASMQ and $33.8 \pm 5.9 \%$ for DHAP group. Only a few patients had HCT of over $40.0 \%$ at CDC Estates clinics on D0. The percentage of cases with mild anaemia (15-29\%) reduced from $5.7 \% \otimes 2.8 \%$ and $6.9 \%$ on day 14 to $0 \%$ on days 28 and 42 , compared to baseline $31.9 \%, 38.9 \%$ and 28.4 respectively in the AL, ASMQ DHAP treatment groups

\section{(2) ADVERSE EVENTS}

The leading adverse reactions recorded during follow up were cough followed by abdominal discomfort, loss of appetite, weakness and pruritus. Overall, 114 of 216 patients complained of cough $62.5 \%$ in the AL group, $47.2 \%$ in the ASMQ group and $58.6 \%$ in the DHAP group. A total of 34 patients reported that they experienced abdominal pains $18.0 \%$ in AL group, 23.6\% in ASMQ group and 5.5\% in DHAP recipients (Table 4). In addition 4 (5.5) reported to experience dizziness and 2 (2.8\%) complained of hallucination only in the ASMQ treatment group. There were blisters in the mouth and a case of serious skin rash in a child by day 35 in the AL treatment although this was not thought to be caused by the drug intake. There were also boils all over the body of a child in ASMQ group at D34 but not due to anti malaria drug intake. All other adverse effects showed no relevant to treatment groups. Patients treated with ASMQ harboured higher frequency of side effect recorded in this study including abdominal pains, body weakness, headache, late vomiting significantly $(P<0.05)$ more frequent than those treated with AL and DHAP $($ Table 4).

Table 4

The frequency of adverse events mild to moderate grades from day 1 to day 42 (related or not) after treatment initiation

\begin{tabular}{|c|c|c|c|c|c|}
\hline \multirow[t]{2}{*}{ Symptomes (N(\%)) } & \multirow[t]{2}{*}{$\mathrm{AL}(\mathrm{N}=72)$} & \multirow[t]{2}{*}{$\operatorname{ASMF}(\mathrm{N}=.72)$} & \multirow[t]{2}{*}{$\operatorname{DHAP}(\mathrm{N}=.72)$} & \multicolumn{2}{|l|}{$P$} \\
\hline & & & & (1) & (2) \\
\hline Weakness/Fatigue & $13 \otimes 18.0 \rrbracket$ & $14 \otimes 19.4 \rrbracket$ & $8(11.1)$ & 0.0080 & 0.0100 \\
\hline Loss of appetite & $12 \otimes 16.7 \rrbracket$ & $14 \otimes 19.4 .0 \rrbracket$ & $4 \nabla 5.5 \rrbracket$ & 0.0130 & 0.0160 \\
\hline Pruritus & $10 \otimes 13.9 \rrbracket$ & $11 \otimes 15.3 \rrbracket$ & $2 \otimes 2.8 \rrbracket$ & 0.0130 & 0.0144 \\
\hline Headache & $2 \otimes 2.8 \rrbracket$ & $9 \rrbracket 12.5 \rrbracket$ & 0 & 0.0030 & 0.0140 \\
\hline Dizziness & 0 & $4 \triangle 5.5 \rrbracket$ & 0 & & 0.0064 \\
\hline Abdominal pain & $13(18.0)$ & $17(23.6)$ & $4 \nabla 5.5 \rrbracket$ & 0.0150 & 0.0210 \\
\hline Vomiting & $8 \otimes 11.1 \rrbracket$ & $15 \otimes 20.8 \rrbracket$ & $3 \otimes 4.2 \rrbracket$ & 0.0100 & 0.0200 \\
\hline Diarrhea & $5 \nabla 6.9 \rrbracket$ & $4 \rrbracket 5.5 \rrbracket$ & $5 \nabla 6.9 \rrbracket$ & & 0.0020 \\
\hline Cough & $45 \rrbracket 62.5 \rrbracket$ & $34 \otimes 47.2 \rrbracket$ & $35 \bowtie 48.6 \rrbracket$ & 0.016 & 0.016 \\
\hline Skin rash & $8 \otimes 11.1 \rrbracket$ & $6 \unrhd 8.3 \rrbracket$ & $2 \rrbracket 2.8 \rrbracket$ & 0.0060 & 0.0060 \\
\hline Hallucination & 0 & $2 \otimes 2.8 \rrbracket$ & 0 & & 0.0032 \\
\hline Insomnia & $3 \rrbracket 4.2 \rrbracket$ & $11 \otimes 15.3 \rrbracket$ & $1 \otimes 1.4 \rrbracket$ & 0.0032 & 0.0160 \\
\hline
\end{tabular}

P value; (1) Compare DHAP vs AL; (2) Compare DHAP vs ASMF

\section{Gametocyte carriage}


A total of $23(10.6 \%)$ patients had gametocytemia at baseline; 6 (8.3\%) were in the AL group, 9 (12.5\%) were in the ASMQ group, and 8 (11.1\%) were in the DHAP group. Of the 193凶89.4\%囚patients without gametocytemia at baseline, $5(15.27 \%)$ in the AL group, 2 (2.7\%) in the ASMQ group, and 3 (4.2\%) in the DHAP group developed gametocytemia post treatment as from D1 and throughout the weeks post treatment (Figure 5). The proportion of cases with gametocytes increased during the first two days of treatment but overall reduced during the first week of follow-up, from $8.3 \%$ to $1.4 \%$ in the group $A L$, from $12.5 \%$ to $0 \%$ in the ASMQ group and from $11.1 \%$ to $1.4 \%$ in the DHAP on day 3. As for DHAP and ASMQ group, all the patients (100\%) cleared their gametocytaemia by day 3. In the AL, ASMQ and DHAP groups, there was no patient with gametocytes at day 7 . At day 28, 2 (2.8\%凶patients in the AL, 1 (1.4 \%) patient in the ASMQ arm and $2(2.8 \%)$ patients in the DHAP arm showed reappearance of gametocytes in their peripheral blood and all of them cleared gametocytaemia by day 35 and 42 .

\section{Discussion}

\section{Clinical and parasitological response}

This study performed a therapeutic Efficacy and safety of AL, ASMQ and DHAP in children with uncomplicated malaria in Cameroon aiming at identifying an alternative treatment regimen to currently used antimalarial. The main outcome of this study was that a 3 days course with AL, ASMQ and AL are all highly effective and well tolerated in the treatment of acute uncomplicated $P$. falciparum malaria in Cameroon. The adequate clinical and parasitological response in AL, ASMQ and DHAP group were $100 \%$ on D7 and D14, consistent with other studies in Cameroon, Ivory Coast and Senegal [27]. On D42, both crude and PCR-corrected ACPRs for DHAP (91.3\% and 100\%) were higher than those recorded in the ASMQ (83.8\% and 97.1\%) and in the AL (81. 1\% and 94.2\%) arms. The PCR-corrected cure rates were generally higher than crude cure rates and were within the ideal anti-malarial drug efficacy range (95\%) recommended by the WHO for 28 days follow-up [35]. The Low uncorrected efficacy has been noted in previous antimalarial efficacy studies [36] and could be explained in part by relatively high malaria transmission. In this study, The overall cure rate of $95.6 \%$ for the $\mathrm{AL}$ arm in a six-dose regimen after a 42-day follow-up is consistent with previous studies [37,38]. But one disadvantage in using AL combination is the problems of two doses per day regimen over three days that may likely reduce compliance and high re-infection rate in areas with high transmission intensity [39]. The ASMQ equally revealed equal efficacy than AL consistent with previous studies in Laos, where ASMQ and AL combinations have been reported to be effective but superior to CQ plus SP in the treatment of uncomplicated falciparum malaria. However, artesunate-mefloquine has been limited by the high cost (3\$ for a single adult treatment), the frequency of adverse effects associated with mefloquine, and the lack of a formulation combining both anti-malarials in a single tablet [23]. A paediatric co-formulation has been developed to improved compliance and been used in Cameroon but has always faced the problem of shortage of stock and was not available nationwide. In addition, reduced efficacy of artesunate-mefloquine has been reported recently from the southeastern border of Thailand [40]. Interestingly, in this study, the highest cure rate was recorded among the DHAP recipient on day 28 and 42. This demonstrates the excellent efficacy and tolerability of DHAP comparable to AL and ASMQ treatment arm, consistent with other findings in Cameroon [17,26,41], Congo , Angola [42], Uganda [39] and Tanzania [43]. Piperaquine tetraphosphate (PQP) is a bisquinoline antimalarial drug that was synthesized in the 1960s at Rhone-Poulenc and independently at the Shanghai Research Institute of Pharmaceutical Industry [44]. Due to the increasing prevalence of chloroquine (CQ)-resistant parasites in southern China, PQP was adopted as the first-line treatment in 1978 [44] and used widely for both prophylaxis and treatment, but its application as monotherapy resulted in the eventual emergence of PQP-resistant parasites, which diminished its use by the late 1980s. Hence piperaquine is now use in combination partner drug, the dihydroartemisinin, a highly active artemisinin derivative and the main in vivo metabolite of artesunate or artemether. Dihydroartemisinin is increasingly use in Africa [39]凶both in 
public and private health facilities and is already part of national treatment recommendations as alternative first-line treatment in Zambian children under 5 years of age[45]. The piperaquine component of DHAP has a longer half-life than lumefantrine, therefore DHAP is likely to provide greater post-treatment prophylaxis and reinfection rates could be prevented using this drug as first-line treatment in children in moderate and high transmission areas [42,46]. Considering the fact that the combination of DHAP is highly effective, its association with any effective partner drug such as trimethoprim will yield a triple combination with excellent therapeutic efficacy and reduced parasite clearance time [47] comparable to DHAP biennial combination. The triple combination will be a promising alternative antimalarial drug that will increased compliance since treatment regimen is reduced to a two days period [27] and will equally provide a prolonged therapeutic lifespan to DHAP biennale combination against multidrug resistance parasites.

\section{Fever and parasite clearance time}

The prolongation of the parasite clearance time has been used as an important early warning sign of reduced artemisinin susceptibility [48] Most ACTs recipient usually cleared their infections within 48hrs of commencing their treatment. In this study, the geometric mean parasite density was reduced by $99.7 \%$ in DHAP group, $99.33 \%$ in ASMQ and 99.22 in the AL group within 24 hours after treatment and completely cleared on day 3 among the 216 patients who completed the treatment. More than $96 \%$ of patients in the DHAP arm were apyrexial 48 hours after treatment compared to $83.5 \%$ in the ASMQ and AL group ( $<$ < 0.001)consistent with previous studies [27] consistent with previous studies where 60 hours to 72 hours was required to completely clear parasites in Vietnamese patients after DHAP intake [48].

\section{Recrudescence or reinfection}

It was observed in the course of this trial, that parasitaemia consisting of young trophozoites appeared on day 28 and 42 in 18 of the 68 ASMQ group, 22 of the 69 AL group and 12 in the 70 DHAP group. The parasitaemia was associated with significant increase in body temperature in $6 ₫ 4$ and 3 patients in AL, ASMQ and DHAP treatment groups respectively. Patients were given paracetamol as antipyretic in addition to quinine as rescue drug. These cases were classified as late treatment failure but, after genotyping most $P$. falciparum recurrences were found to be caused by reinfection. Thus, 6 cases in AL, 2 in ASMQ and 2 in DHAP treatment group were attributed to recrudescence of residual parasitemia and the others were attributed to new infections, this observation is similar to observation made in other malaria endemic countries $[36,46,49]$. The rate of true recrudescence was less than $5 \%$ in all the treatment groups. The high reinfection rates observed in this study, comparable to recrudescence, is an indication of a very high malaria transmission in this study area. This study therefore advocates for a continuous use of long lasting insecticide treated nets for malaria prevention alongside with an effective and well tolerated ACT such as DHAP for an effective malaria control in meso to high malaria transmission areas .

\section{Safety and Tolerability}

In this study, there was no report of serious adverse events and none of the patients followed-up reported any serious drug-related side effects following the administration of AL, ASMQ and DHAP. Some mild adverse events such as abdominal discomfort, loss of appetite, weakness were noted, but these events resolved spontaneously and gave no reason to stop the treatment in either treatment arm. Importantly, the most frequent adverse effect reported in all the treatment groups was cough but it is difficult to determine whether this side effect was related to antimalarial drugs intake or to the resolving symptoms of malaria. However, all the treatments were well tolerated and safe, the drug related adverse events were transient and of mild or moderate intensity. 
In this study, the frequency of reporting of loss of appetite, late vomiting, abdominal pain, insomnia and pruritus, were higher in the AL and ASMQ arms than in the DHAP arm. Drug toxicity of mefloquine and drug-related adverse events have been numerously reported. Mefloquine has been linked with adverse events of gastrointestinal and central nervous system origin [47,50]. Gastrointestinal and nervous system adverse events, similar to those experienced in this trial, have often been described after intake of mefloquine. Likewise, in some clinical trials, mefloquine recipients reported a higher rate of adverse effects compared to chloroquine, halofantrine, and artemether-lumefantrine recipients. In addition, mefloquine treatment in patients of all ages on the Thai-Burmese border revealed drug-induced vomiting, followed by dose-related anorexia, nausea, late vomiting, and dizziness. However, drug-induced vomiting was reduced when ASMQ was administer as fixed dose combination as compare with loose tablets [51].

It was observed in the course of this study that AL, ASMQ and DHAP harboured similar impact in the improvement of Haematocrit value during treatment. There was a clear increase in the average haemoglobin levels in the three treatment groups during the 42 days of follow-up These findings confirmed the action of ACT in haemoglobin recovery as stated in previous study [52]. In this study, apart from the rapid clearance of asexual forms of $P$. falciparum, there was a significant reduction in gametocyte count. The data revealed that these drugs ultimately cleared gametocyte from peripheral blood. This shows that these ACTs exhibits considerable gametocidal effect. Thus, the three antimalarial drugs were effective in treatment of uncomplicated malaria in children. The overall efficacy and tolerability of AL are similar to those of ASMQ but AL in Cameroon is sold directly over the counter, therefore its useful therapeutic life might be compromised sooner following widespread use. Although the DHAP is highly effective for the treatment of uncomplicated malaria, its high cost may be a limitation to a large scale deployment rendering this drug affordable mostly by individuals with higher income in Cameroon. The wholesale price for a complete adult dose in Laborex is 3000 XAF which is approximately 6 USD whereas in the global market DHAP remain less expensive comparable to other ACTs. The overall cost for a complete adult dose is 1 USD [15,53] . Overall, the problem of availability and affordability of these ACT are a cause of concern in Cameroon added to unspecific treatment of all fever patients with these ACTs in most of hospital settings in Cameroon and in Africa in general $[5,54,55,56]$. The principle behind is that, most health workers perceived that malaria is endemic in Africa and deemed it relevant to treat all fever cases as malaria even with a malaria negative microscopic slide [5,56]. This over consumption of ACT may reduce its efficacy, even in areas where resistance to this drug have not yet been reported. It is therefore important to constantly monitor the efficacy of these drugs to enable the NMCP to adjust on time the therapeutic scheme of malaria case management.

\section{Conclusion}

The ACTs including DHAP, AL, and ASMQ have shown to be effective and safe in the treatment of Plasmodium falciparum malaria in Cameroon. The anti-gametocidal effect of DHAP, its highest cure rate, the difference in recrudescence and fewer side effects recorded in this study indicate that DHAP may be the preferred antimalarial treatment that will play an important role in the reduction of malaria transmission added to its advantage of being available for single dosage regimen in a fixed-dose co-formulations, which improves compliance. If subsidized in each country, then DHAP could be an alternative first-line treatment for an effective management of malaria in Cameroon and in malaria endemic countries.

\section{Abbreviations}

ACT, artemisinin-based combination therapy; 
$\mathrm{AL}$, artemether - lumefantrine

ASMQ, artesunate - mefloquine

DHAP, dihydroartemisinin-piperaquine;

ETF, early treatment failure

LTF, late treatment failure

LPF, late parasitological failure

LLIN, long-lasting insecticidal net;

\section{Declarations}

\section{Acknowledgements}

Local support was provided by the physicians and nurses of CDC clinics and hospitals in Cameroon. The authors would like to thank all study participants and their parents/guardians and the study team members in each clinics and health facilities for their participation.

\section{Authors' contributions}

MTN, JHC and XNZ conceived and designed the study. XNZ and SMR coordinated the study. SMR, MTN and PFG supervised enrollment and patients follow-up and data collection by the study teams. MTN and ET performed the molecular analysis supervised by JHC. MTN, PFG and ET prepared the manuscript. SMR and JHC reviewed the manuscript. XNZ critically revised the manuscript. All authors read and approved the final manuscript.

\section{Funding}

This study was funded by the National Institute of Parasitic Disease, China CDC, and Shanghai, supported by Ministry of Science and Technology (grant No. 2012ZX10004-220), People's Republic of China. The National Institute of Parasitic Disease, provided platform for molecular analysis. Financial support was also provided by the Chinese National Science and Technology Major Project (grant No. 2012ZX10004-220), China UK Global Health Support Programme (grant no. GHSP-CS-OP1), and International Collaboration Project from the Ministry of Science and Technology (grant No. 2010DF33970).

\section{Availability of data and materials}

The dataset used and analysed from this study is available from the corresponding author

\section{Ethics approval and consent to participate}

This study was approved by the National ethic committee of Cameroon and by IRB of the National Institute of Parasitic Diseases, Chinese Centre for Disease Control and Prevention. Each, participant was informed of the aims, methods, anticipated benefits and potential hazards of the study. Then, informed, written consent was obtained by the investigator from every parent/guardian of patients participating in the study. The subject was informed that he/she was at liberty to abstain from participation in the study and that he/she was free to withdraw the consent of participation at any time. Participation fee for all patients (transportation, laboratory analysis, treatment) was free of 
charge. The trial is registered with controlled-trials.com in the website:

https://clinicaltrials.gov/ct2/show/NCT02974348 under the registration number NCT02974348

\section{Consent for publication}

Not applicable

\section{Competing interests}

The authors declare that they have no competing interests.

\section{Reference}

1. Murray MJL, Rosenfeld LC, Lim SS, Andrews KG, Foreman KJ, Haring D, Fullman N, Naghavi M, Lozano R, Lopez AD, 2012. Global malaria mortality between 1980 and 2010: a systematic analysis. The Lancet, Volume 379, Issue 9814, Pages 413 - 431.

2. WHO. World malaria report 2018. Geneva: World Health Organization; 2018.

3. NMCP. National Malaria Control Program (NMCP) report, 2012. Cameroon: Ministry of Public Health; 2012.

4. Staedke SG, Kamya MR,Dorsey G, Gasarina A, Neleezi G, Edwin D, Charle bois, Thee P. J. R. (2004). Amodiaquine, sulfadoxine-pyriméthamine, and combination therapy for treatment of uncomplicated malaria in Kampala, Uganda : a randomised trial. The lancet vol. $368: 368-374$.

5. Mangham L J, Cundill B, Achonduh O A, Ambebila J N, Lele A K, Metoh T N, Ndive S N, Ndong I C, Nguela RL, Nji AM, Orang-Ojong B, Pamen-Ngako J, Wiseman V and Mbacham W F, 2012. Malaria prevalence and treatment of febrile patients at health facilities and medicine retailers in Cameroon. Tropical Medicine \& International Health, Volume 17, Issue 3,pages 330-342.

6. Same-Ekobo A, Basco L \& Metoh T, 2007. Mise au point sur l'épidémiologie du paludisme en Afrique centrale avec les schémas thérapeutiques recommandés par les PNLP. Bull Soc Pathol Exot,2007,100, 1, 65-93

7. Ringwald P, Keundjian A, Same Ekobo A and Basco LK. Chimiorésistance de falciparum en milieu urbain à Yaoundé, Cameroun. Part 2: Evaluation de l'efficacité de l'amodiaquine et de l'association sulfadoxinepyriméthamine pour le traitement de l'accès palustre simple à Plasmodium falciparum à Yaoundé, Cameroun. Trop. Med. Int. H.;2000, 5 (9) : 620-627.

8. Soula G, Taptue Fotso JC, Foumane V, Same Ekobo A, Kollo B, 2002. Description épidémiologique et conséquences économiques de la rougeole et du paludisme dans une entreprise agro-industrielle du Sud Cameroun. Bull. liais. doc. OCEAC: 35 (2) : 35-44.

9. Basco LK, Samé Ekobo A, Foumane V, Ndounga M, Metoh TN, Ringwald P, and Soula G, 2002. Therapeutic efficacity of sulfadoxine-pyrimethamine, amodiaquine and sulfadoxine -pyriméthamine- amodiaquine combination of the treatment of uncomplicated Plasmodium falciparum malaria in Young children in Cameroun. W. H. O., 80: 538-545

10. Metoh N T, Tahar, R, Same-Ekobo A, Foumane Ngane V, Soula G, Basco LK, 2010. Molecular Epidemiology of Malaria in Cameroon XXIX. Characterization of DHFR and Drug Resistance Markers and Efficacy of SulfadoxinePyrimethamine Monotherapy in Children in Niete (HEVECAM),Vol 2 (1),p146-152.

11. Mbacham W, Evehe M, Mbulli A, Akaragwe I, Tawe B, Djoko C, Gang B, Masumbe P, Mokube A, Atogho B, Ebeng R, 2005a. Therapeutic efficacy of Sulfadoxine-Pyrimethamine (Fansidar ${ }^{\circledR}$ ) and mutation rates to Anti-folate genes in different regions of Cameroon. Acta Trop, 95S:337. 
12. Zwang J., Olliaro P., Barennes H, Bonnet M, Brasseur P, Bukirwa H, Cohuet S, D'Alessandro U, Djimdé A, Karema C, Guthmann J-P, Hamour S, Ndiaye J-L, Mårtensson A, Rwagacondo C, Sagara I, Same-Ekobo A, Sirima SB, van den Broek I, Yeka A, Taylor W RJ, Dorsey G and Randrianarivelojosia M. Efficacy of artesunate-amodiaquine for treating uncomplicated falciparum malaria in sub-Saharan Africa: a multi-centre analysis. Malaria Journal 2009, 8:203 doi:10.1186/1475-2875-8-203

13. Silachamroon U, Krudsood S, Thanachartwet W, et al. An open, randomized trial of three-day treatment with artesunate combined with a standard dose of mefloquine divided over either two or three days, for acute, uncomplicated falciparum malaria. Southeast Asian JTrop Med Public Health 2005; 36: 591-6.

14. Ashley EA, McGready R, Hutagalung R et al. (2005) A randomized, controlled study of a simple, once-daily regimen of dihydroartemisinin-piperaquine for the treatment of uncomplicated, multidrug-resistant falciparum malaria. Clinical Infectious Diseases 41, 425-432.

15. Mutabingwa T.K. (2005). Artemisinin-based combination therapies (ACTS): Best hope for malaria treatment but inaccessible to the needy!. Acta tropica 95, 305-315.

16. Moyou SR, Kengne L, Chinwengozi M, Chendjou P. Efficacité et tolérance de la combinaison artesunate + amodiaquine pour le traitement du paludisme simple dans le District de Sante de NDOP, Province du Nord-Ouest, Cameroun, 2007. Report to Ministry of Health.

17. Whegang1 SY, Tahar R, Foumane VN, Soula G, Gwét H, Thalabard J-C, Basco LK (2010). Efficacy of nonartemisinin- and artemisinin-based combination therapies for uncomplicated falciparum malaria in Cameroon. Malaria Journal 9:56, 1-10.

18. World Health Organization. World Malaria Report 2013(2013).

19. Yavo W, Faye B, KueteT, Djohan V, Oga SA, Kassi RR, Diatta M, Ama M V, Tine R, Ndiaye J-L, Evi1J-B, Same-Ekobo A, Faye $\mathrm{O}$ and Koné $\mathrm{M}, 2011$. Multicentric assessment of the efficacy and tolerability of dihydroartemisininpiperaquine compared to artemether-lumefantrine in the treatment of uncomplicated Plasmodium falciparum malaria in sub-Saharan Africa. 10:198 http://www.malariajournal.com/content/10/1/198

20. Whegang YS, Chiabi A, Basco L K. Monitoring the Efcacy and Safety of Artemisinin-Based Combination Therapies: A Review and Network Meta-analysis of Antimalarial Therapeutic Efcacy Trials in Cameroon. Drugs in R\&D (2019) 19:1-14 https://doi.org/10.1007/s40268-018-0259-3

21. Mbacham WF, Njuabe MT, Evehe MS, Moyou R, Same Ekobo A, (2005b) Antimalarial drug studies in Cameroon reveal deteriorating fansidar and amodiaquine cure rates. Malaria research and control in Cameroon.. Cam. Acad. Sci., 5 : 58-63.

22. Denis MB, Davis TM, Hewitt S, Incardona S, Nimol K, Fandeur T, Poravuth Y, Lim C, Socheat D: Efficacy and safety of dihydroartemisinin-piperaquine (Artekin) in Cambodian children and adults with uncomplicated falciparum malaria. Clin Infect Dis 2002, 35:1469-1476.

23. Smithuis F, Kyaw MK, Phe O, Aye KZ, Htet L, Barends M, Lindegardh N, Singtoroj T, Ashley E, Lwin S, Stepniewska $\mathrm{K}$, White NJ: Efficacy and effectiveness of dihydroartemisinin-piperaquine versus artesunatemefloquine in falciparum malaria: an open label randomized comparison. Lancet 2006, 367:2075-2085.

24. Fanello Cl, Karema C, van Doren W, van Overmeir C, Ngamije D, D’Alessandro U: A randomised trial to assess the safety and efficacy of artemether-lumefantrine (Coartem $\left.{ }^{\circledR}\right)$ for the treatment of uncomplicated Plasmodium falciparum malaria in Rwanda. Trans R Soc Trop Med Hyg 2007, 101:344-350.

25. Piola P, Fogg C, Bajunirwe F, Biraro S, Grandesso F, Ruzagira E, Babigumira J, Kigozi I, Kiguli J, Kyomuhendo J, Ferradini L, Taylor W, Checchi F, Guthmann JP: Supervised versus unsupervised intake of six-dose 
artemetherlumefantrine for treatment of acute, uncomplicated Plasmodium falciparum malaria in Mbarara, Uganda: a randomised trial. Lancet 2005, 365:1467-1473.

26. Nji AM, Ali IM, Moyeh MN, Ngongang EO, Ekollo AM, Chedjou JP. Randomized non-inferiority and safety trial of dihydroartemisin-piperaquine and artesunate-amodiaquine versus artemetherlumefantrine in the treatment of uncomplicated Plasmodium falciparum malaria in Cameroonian children. Malar J. 2015;14:27

27. Menan H, Faye O, Same-Ekobo A, Oga ASS , Faye B , Barro CPK, Kuete T , N'diaye J-L , Ama-Moor V, Tine R, Yavo W , Kane D, Kassi KF, Kone M (2011). Comparative study of the efficacy and tolerability of dihydroartemisininpiperaquine-trimethoprim versus artemether-lumefantrine in the treatment of uncomplicated Plasmodium falciparum malaria in Cameroon, Ivory Coast and Senegal. Malaria Journal. 01/2011; 10:185. DOI: 10.1186/1475-2875-10-185

28. Fairhurst R M. and Dondorp A M Artemisinin-resistant Plasmodium falciparum Published in final edited form as: Microbiol Spectr. 2016 June ; 4(3): doi:10.1128/microbiolspec.El10-0013-2016.

29. Dondorp, A.M.; Nosten, F.; Yi, P.; Das, D.; Phyo, A.P.; Tarning, J.; Lwin, K.M.; Ariey, F.; Hanpithakpong, W.; Lee, S.J.; Ringwald, P.; Silamut, K.; Imwong, M.; Chotivanich, K.; Lim, P.;Herdman, J.; An, S.S.; Yeung, S.; Singhasivanon, P.; Day, N.P.; Lindegardh, N.; Socheat, D.Artemisinin resistance in Plasmodium falciparum malaria. Engl. hJ. Med. 2009, 361, 455-67.

30. Wanji S, Tanke T, Atanga SN, Ajonina C, Nicolas T, Fontenille D: Anopheles species of the Mount Cameroon region: biting habit, feeding behaviour and entomological inoculation rates. Trop Med Int Health 2003, 8:643649.

31. Bigoga JD, Manga L, Titanji VPK, Coetzee M, Leke RGF. Malaria vectors and transmission dynamics in coastal south-western Cameroon. Malar J. 2007;6:5-17. doi: 10.1186/1475-2875-6-5.

32. WHO. Assessment and monitoring of antimalarial drug efficacy for the treatment of uncomplicated falciparum malaria (WHO/HTM/RBM/2003.50) (http://www.who.int/malaria/resistance). Geneva, World Health Organization, 2003.

33. WHO. Methods and techniques for clinical trials on antimalarial drug efficacy: genotyping to identify parasite populations - world health organization whqlibdoc.who.int/publications/2008/9789241596305_eng.pdf

34. FDA, 1996. Good Clinical Practice Guidances. Accessed on November 28, 2006, http://www.fda.gov/cder/guidance/959fnl.pdf.

35. Mendis K: WHO guidelines for the treatment of malaria: Implication for the next generation of antimalarial medicines. Press release Global Malaria Programme, Washington

36. Plucinski MM, Dimbu PR, Macaia AP, Ferreira CM, Samutondo C, Quivinja J, et al. Efficacy of artemetherlumefantrine, artesunate-amodiaquine, and dihydroartemisinin-piperaquine for treatment of uncomplicated Plasmodium falciparummalaria in Angola, 2015. Malar J. 2017;16:62. doi: 10.1186/s12936-0171712-4.

37. Haque R, Thriemer K, Wang Z, Sato K, Wagatsuma Y, Salam MA, Akther S, Akter J, Fukuda M, Miller RS and Noedl $\mathrm{H}$. Short report: Therapeutic efficacy of artemether-lumefantrine for the treatment of uncomplicated plasmodium falciparum malaria in Bangladesh, J. Trop. Med. Hyg., 76(1), 2007, pp. 39-41.

38. Sagara I, Rulisa S, Mbacham W, Adam I, Sissoko K, Maiga H, Traore OB, Dara N, Dicko YT, Dicko A, Djimdé A, g Jansen $\mathrm{F} \mathrm{H}$, and Doumbo OK. Efficacy and safety of a fixed dose artesunate-sulphamethoxypyrazinepyrimethamine compared to artemether-lumefantrine for the treatment of uncomplicated falciparum malaria across Africa: a randomized multi-centre trial. Malaria Journal 2009, 8:63 doi:10.1186/1475-2875-8-63 
39. Kamya MR, Yeka A, Bukirwa H, Lugemwa M, Rwakimari JB, Staedke SG, Talisuna AO, Greenhouse B, Nosten F, Rosenthal PJ, Wabwire-Mangen F, Dorsey G: Artemether-lumefantrine versus dihydroartemisinin-piperaquine for treatment of malaria: a randomized trial. PLoS Clin Trials 2007, 2(5):e20

40. Vijaykadga S, Rojanawatsirivej C, Cholpol S, Phoungmanee D, Nakavej A, Wongsrichanalai C. In vivo sensitivity monitoring of mefloquine monotherapy and artesunate-mefloquine combinations for the treatment of uncomplicated falciparum malaria in Thailand in 2003. Trop Med Int Health 2006;11:211-9.

41. Whegang Youdom S, Tahar R, Basco LK. Comparison of antimalarial drug efcacy in the treatment of uncomplicated malaria in African children and adults using network meta-analysis. Malar J. 2017;16:311

42. Okell, L. C. et al. Contrasting benefits of different artemisinin combination therapies as first-line malaria treatments using model-based cost-effectiveness analysis. Commun.5:5606 doi: 10.1038/ncomms6606 (2014).

43. Kabanywanyi AM, Mwita A, Sumari D, Mandike R, Mugittu K and Abdulla S. Efficacy and safety of artemisininbased antimalarial in the treatment of uncomplicated malaria in children in southern Tanzania Malaria Journal, 2007

44. Davis, TM, Hung T Y, Sim IK, Karunajeewa HA, and llett KF. Piperaquine: a resurgent antimalarial drug. Drugs 65:75-87

45. Nambozi M, Van Geertruyden JP, Hachizovu S, Chaponda M, Mukwamataba D, Mulenga M, et al. Safety and efcacy of dihydroartemisinin-piperaquine versus artemether-lumefantrine in the treatment of uncomplicated Plasmodium falciparum malaria in Zambian children. Malar J. 2011; $10: 50$.

46. Davlantes E,_. DimbuPR, FerreiraCM, JoaoMF, Pode et al. Efficacy and safety of artemether-lumefantrine, artesunate-amodiaquine, and dihydroartemisinin-piperaquine for the treatment of uncomplicated Plasmodium falciparum malaria in three provinces in Angola, 2017. Malar J. 2018; 17: 144.

47. Gargano N, Ubben D, Tommasini S, Bacchieri A, Corsi M, Bhattacharyya PC, Rao B. HK, Dubashi N, Dev V, Ghosh SK, Kumar A, Srivastava B and Valecha N. Therapeutic efficacy and safety of dihydroartemisinin-piperaquine versus artesunate-mefloquine in uncomplicated Plasmodium falciparum malaria in India. Malaria Journal 2012, 11:233 http://www.malariajournal.com/content/11/1/233

48. Thanh N X, Trung TN, Phong NC, Quang HH, Dai B, Shanks G D, Chavchich M and D Edstein M. The efficacy and tolerability of artemisininpiperaquine (ArtequickW) versus artesunateamodiaquine (Coarsucam ${ }^{\mathrm{TM}}$ ) for the treatment of uncomplicated Plasmodium falciparum malaria in south-central Vietnam. Malaria Journal 2012, 11:217 http://www.malariajournal.com/content/11/1/217

49. Falade $C^{1}$, Makanga M, Premji Z, Ortmann CE, Stockmeyer M, de Palacios PI. Efficacy and safety of artemetherlumefantrine (Coartem) tablets (six-dose regimen) in African infants and children with acute, uncomplicated falciparum malaria. Trans R Soc Trop Med Hyg.2005 Jun;99(6):459-67.

50. Schlagenhauf P, Adamcova M, Regep L, Schaerer MT, Bansod S, Rhein HG: Use of mefloquine in children - a review of dosage, pharmacokinetics and tolerability data. Malar J 2011, 10:292.

51. Wells S , Diap G, and Kiechel J-R. The story of artesunate-mefloquine (ASMQ), innovative partnerships in drug development: case study.Malar J. 2013; 12: 68.

52. Van den Broek I, Kitz C, Al Attas S, Libama F, Balasegaram M and Guthmann J-P. Efficacy of three artemisinin combination therapies for the treatment of uncomplicated Plasmodium falciparum malaria in the Republic of Congo. Malaria Journal 2006, 5:113 doi:10.1186/1475-2875-5-113

53. Hien TT, Dolecek C, Mai PP. Dihydroartemisininpiperaquine against multidrug-resistant Plasmodium falciparum malaria in Vietnam: randomized clinical trial. Lancet 2004; 363: 18-22. 
54. Achonduh OA, Mbacham W F, Mangham-J L, Cundill B, Chandler C, Pamen-NJ., Lele AK, Ndong1 I C, Ndive SN, Ambebila JN, Orang-Ojong BB, Metoh TN, Akindeh- N M and Wiseman V. Designing and implementing interventions to change clinicians' practice in the management of uncomplicated malaria: lessons from Cameroon. Malaria Journal 2014 13:204.

55. Gosling RD, Drakeley CJ, Mwita A, and Chandramohan D . Presumptive treatment of fever cases as malaria: help or hindrance for malaria control? Malar J. 2008; 7: 132.

56. Bruxvoort KJ, Leurent B, Chandler CIR, Ansah EK, Baiden F, Bjorkman A, et al. The impact of introducing malaria rapid diagnostic tests on fever case management: a synthesis of ten studies from the ACT Consortium. Am J Trop Med Hyg. 2017;97:1170-9.

\section{Figures}




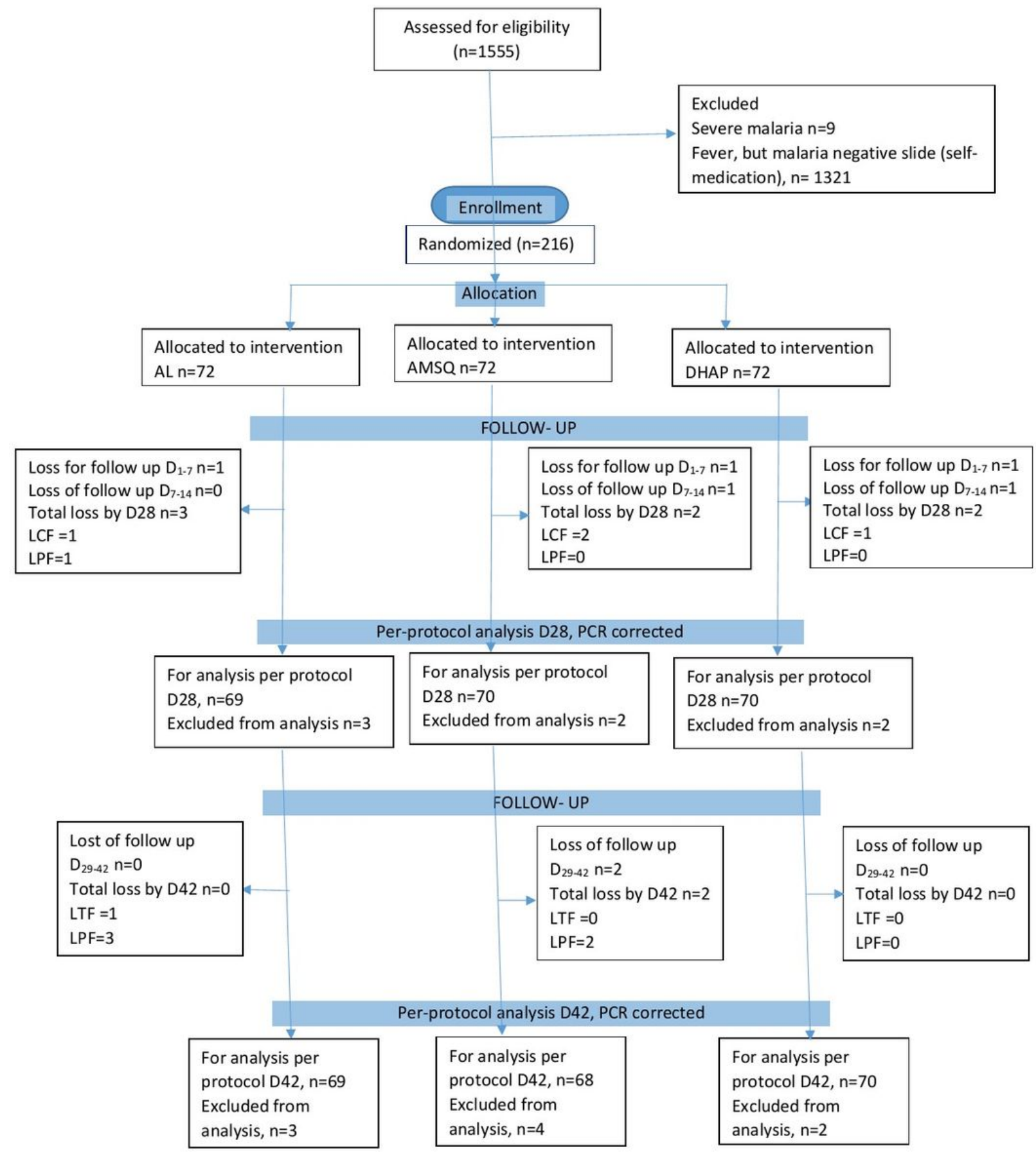

Figure 1

Study profile 


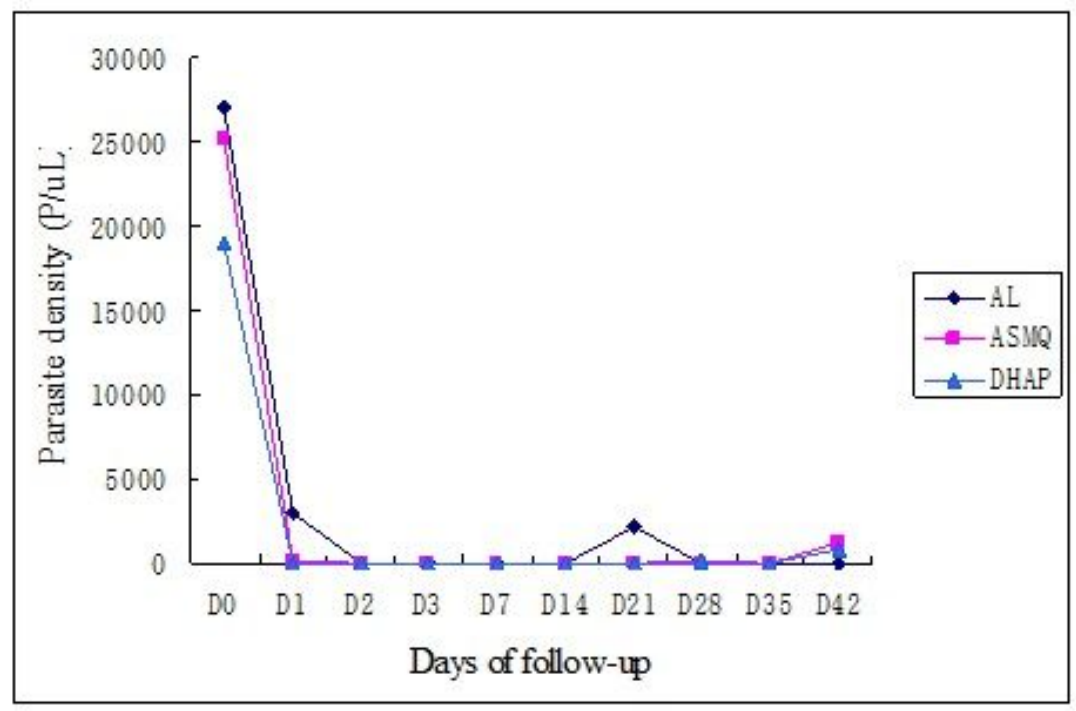

Figure 2

Parasite clearance profile relative to days of follow up

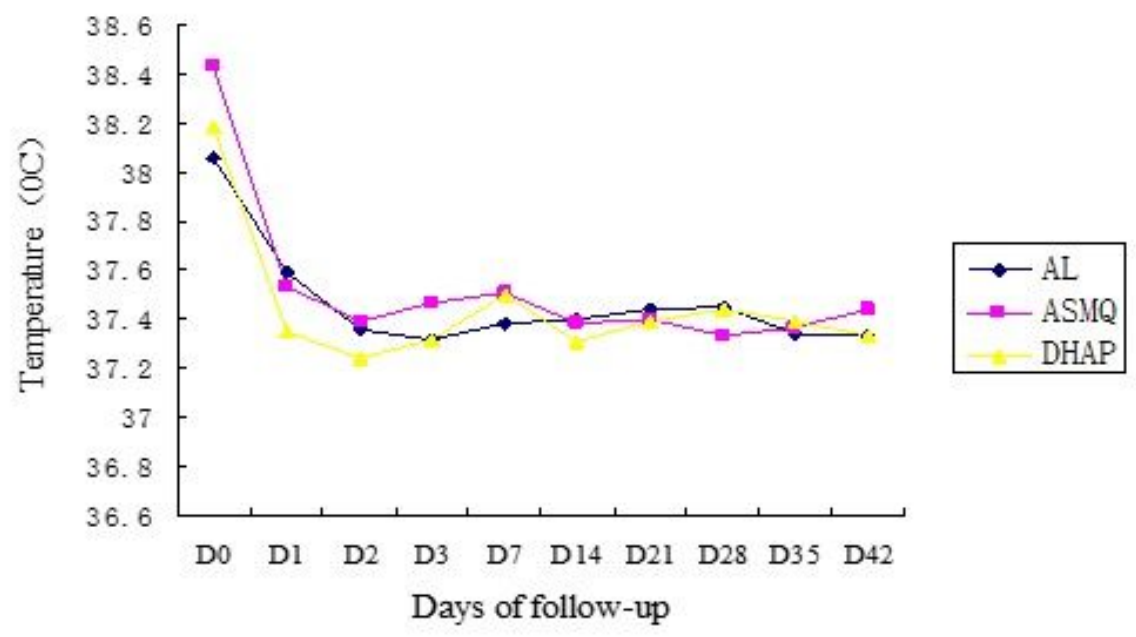

Figure 3

Trends of mean temperature per treatment groups relative to days of follow up 




Figure 4

Hematocrit values measured at 2-weekly visits relative to treatment groups

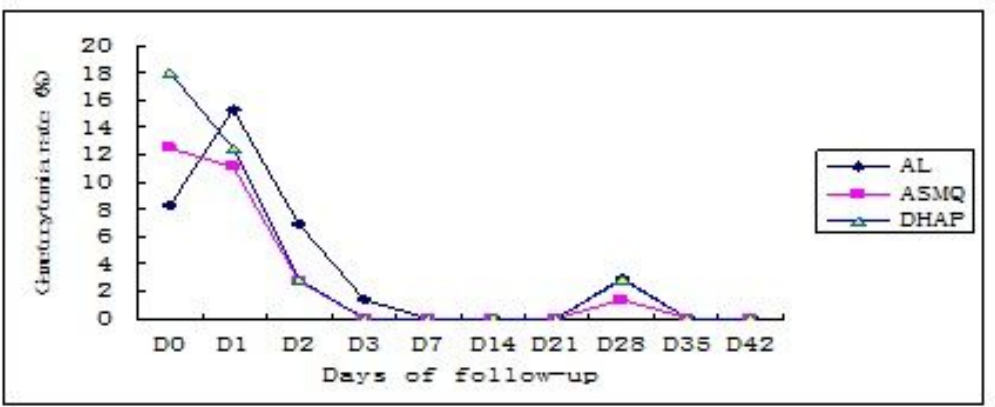

Figure 5

Gametocytemia per treatment group during follow-up. 